\title{
In-plane noncollinear exchange coupling mediated by helical edge states in quantum spin Hall systems
}

\author{
Jinhua Gao, ${ }^{1}$ Weiqiang Chen, ${ }^{1}$ X. C. Xie, ${ }^{2,3}$ and Fu-chun Zhang ${ }^{1}$ \\ ${ }^{1}$ Department of Physics and Center of Theoretical and Computational Physics, The University of Hong Kong, Hong Kong, China \\ ${ }^{2}$ Beijing National Laboratory for Condensed Matter Physics and Institute of Physics, Chinese Academy of Sciences, Beijing 100 190, China \\ ${ }^{3}$ Department of Physics, Oklahoma State University, Stillwater, Oklahoma 74078, USA
}

(Received 25 July 2009; revised manuscript received 7 October 2009; published 3 December 2009)

\begin{abstract}
We study the Ruderman-Kittel-Kasuya-Yoshida interaction mediated by helical edge states in quantum spin Hall system. The helical edge states induce an in-plane noncollinear exchange coupling between two local spins, in contrast to the isotropic coupling induced in normal metal. The angle between the two local spins in the ground state depends on the Fermi level. This property may be used to control the angle of spins by tuning the electric gate.
\end{abstract}

DOI: $10.1103 /$ PhysRevB.80.241302

PACS number(s): 73.43.-f, 75.30.Hx, 72.25.Dc, 85.75.-d

\section{INTRODUCTION}

Recently, the study of quantum spin Hall (QSH) state has attracted much attentions ${ }^{1-5}$ for its interesting topology and for its potential applications in the field of spintronics.

The well-known examples of the topologically nontrivial states are the integer and fractional quantum Hall states, where the quantization of the Hall conductance is protected by a topological invariant. The QSH insulator is a topologically insulating phase with time-reversal symmetry. A twodimensional QSH insulator has a charge excitation gap in the bulk and gapless helical edge states. Two states with opposite spin polarization counterpropagate at a given edge. ${ }^{1,4}$ Due to the time-reversal symmetry, these spin-filtered edge states are stable against weak interaction and disorder.,6 Hence, they can be viewed as effective spin and charge conducting channels and be used to construct promising spintronic devices with low power consumption.

Ruderman-Kittel-Kasuya-Yoshida (RKKY) interaction is an effective interaction between two local spins mediated by conduction electrons. ${ }^{7}$ It plays an important role in many fields of solid-state physics, e.g., giant magnetoresistance ${ }^{8}$ and dilute magnetic semiconductor. ${ }^{9}$ More recently, people propose that the controllable RKKY interaction can be used to manipulate the quantum states of the local spins, which is a crucial point for the spintronics and quantum computing. ${ }^{10,11}$ Thereafter, the RKKY interactions in different spintronic materials, e.g., spin-orbital system ${ }^{12-14}$ and graphene, ${ }^{15-18}$ have been investigated carefully in order to facilitate the further development of the spintronic devices. Compared with the normal cases, the effective interactions between local spins in these systems exhibit rather different properties. The RKKY interaction in the spin-orbital system becomes a twisted exchange coupling since it is a spindependent system. ${ }^{12-14}$ The spin polarizations of the two local spins are no longer collinear in this case. As for the graphene, due to its special electronic band dispersion, it is found that the RKKY interaction is ferromagnetic for local spins within equivalent sublattices but antiferromagnetic for opposite sublattices when the Fermi level is near the Dirac point. 15,16

Being a spintronic material, it is a quite intriguing and practical problem that what the RKKY interaction in the
QSH insulator is. Since the helical edge states are the only conducting channels in this system, the problem becomes that what the exchange interaction mediated by the helical edge states is.

In this Rapid Communication, we investigate the RKKY interaction mediated by the helical edge states in the QSH insulator. Our theoretical analysis is mainly based on the simplified model of the helical edge state, $, 2,4$ in which the spin of the carriers is assumed to be parallel or antiparallel along the $z$ axis, as shown in Fig. 1. Actually, it is proposed that the QSH insulator could be realized in various kinds of systems, e.g., graphene, ${ }^{1}$ quantum well, ${ }^{2,3}$ or semiconductor materials with special strain gradient. ${ }^{4,5}$ The descriptions of the helical edge state in different systems may be different. However, the model we used is the simplest and most basic one. It grasps the primary characteristic of the helical states, i.e., the correlation between the spin polarization and propagation of the carrier, and also is the exact expression of the helical edge states in the semiconductor system with special strain gradient. Hence, we believe that this model is a good starting point for analyzing of the RKKY interaction mediated by the helical edge state.

We find that the helical property and the linear dispersion of the edge conducting electrons will lead to an in-plane and

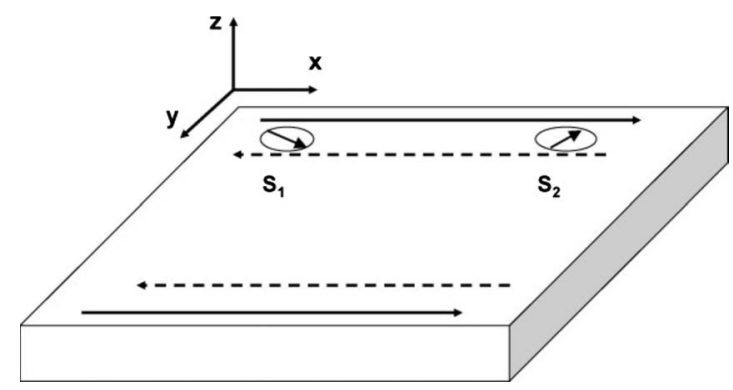

FIG. 1. Schematic of the RKKY interaction between two local spins mediated by the helical edge states in QSH insulator. The QSH insulator is assumed to be a finite strip geometry which is infinite along the $x$ direction. Two local spins $S_{1}$ and $S_{2}$ are located along one edge of the QSH insulator. The solid (dashed) line represents the up-spin right movers (down-spin left movers) of the helical edge states on this edge. 
noncollinear exchange coupling between two local spins along the edge. In the asymptotic limit, this interaction has a simple expression. We can see that the angle between the two spins can be controlled by adjusting the Fermi energy of the system. When the Fermi surface is near the Dirac point, i.e., the Fermi energy is near zero, the effective interaction becomes a constant antiferromagnetic exchange coupling. Actually, due to the helicity, pure spin current can be achieved in these edge states. Hence, this effective coupling can also be viewed as RKKY interaction mediated by pure spin current.

This paper is organized as follows. In Sec. II, the RKKY interaction mediated by the helical edge states of QSH insulator is derived. In Sec. III, we discuss the special properties of this exchange interaction. Finally, a brief summary will be given in Sec. IV.

\section{MODEL AND FORMALISM}

We consider the simplest model of helical edge states, which has been successfully used in the study of the tunneling properties of the helical edge states in the QSH insulator. ${ }^{4,19,20}$ The schematic is shown in Fig. 1. The helicity correlates the spin polarization with the propagation. Here, we assume that the right (left) movers $\psi_{R \uparrow}\left(\psi_{L \downarrow}\right)$ carry spin up (down). In the noninteracting case, the linearized Hamiltonian is

$$
H_{0}=-v_{F} \int d x\left(\psi_{R \uparrow}^{+} i \partial_{x} \psi_{R \uparrow}-\psi_{L \downarrow}^{+} i \partial_{x} \psi_{L \downarrow}\right),
$$

where $v_{F}$ is the Fermi velocity.

The localized spins are magnetic impurities and are denoted by $S_{1}$ and $S_{2}$. Normally, the Kondo coupling between the local moments and conducting electrons are

$$
H_{1}=-\frac{J}{2} \sum_{\substack{i=1,2 \\ \alpha, \beta=\uparrow, \downarrow}} \sigma_{\alpha \beta} S_{i} \int d x \psi_{\alpha}^{+}(x) \delta\left(x-x_{i}\right) \psi_{\beta}(x),
$$

where $J$ is the coupling constant, $\sigma_{\alpha \beta}$ is the spin operator of the conducting electrons. $\alpha$ and $\beta$ are the spin indices. $i=1,2$ is the index of the local spins since in order to study the RKKY interaction, we need to consider two local spins. For the helical edge states, the only difference is that the spin polarization is correlated with the propagation. Hence, in the Kondo coupling expression of the helical edge states, $\psi_{\uparrow}(x)\left[\psi_{\downarrow}(x)\right]$ means $\psi_{R \uparrow}(x)\left[\psi_{L \downarrow}(x)\right] .^{4,21}$

If the coupling $J$ is small, $H_{1}$ can be treated as a perturbation on $H_{0}{ }^{22}$ The RKKY interaction between two local spins $S_{1}$ and $S_{2}$ can be calculated from the second-order perturbation theory ${ }^{12,14,23}$

$$
\begin{aligned}
E_{R K}= & -\frac{J^{2}}{\pi} \operatorname{Im} \int_{-\infty}^{E_{F}} d \omega \\
& \times \operatorname{Tr}\left[\left(S_{1} \cdot \sigma\right) G^{r}\left(R_{12} ; \omega\right)\left(S_{2} \cdot \sigma\right) G^{r}\left(-R_{12} ; \omega\right)\right],
\end{aligned}
$$

where $E_{F}$ is the Fermi energy, $R_{12}=x_{1}-x_{2}$ is the distance between the two local spins, and Tr means the trace over the spin degree of freedom of conduction electrons. Therefore, the study of RKKY interaction has been reduced to the calculation of the retarded Green's function of the helical edge states.

The definition of retarded Green's function is

$$
G_{\alpha \beta}^{r}\left(x t, x^{\prime} t^{\prime}\right)=-i \theta\left(t-t^{\prime}\right)\left\langle\left\{\psi_{\alpha}(x t), \psi_{\beta}^{+}\left(x^{\prime} t^{\prime}\right)\right\}\right\rangle
$$

where $\alpha, \beta=\{R \uparrow, L \downarrow\}$ are the helical spin indices. Because that there is no coupling between different spins in the noninteracting Hamiltonian (1), the nonzero Green's functions are $G_{R \uparrow R \uparrow}^{r}\left(x, x^{\prime}\right)$ and $G_{L \downarrow L \downarrow}^{r}\left(x, x^{\prime}\right)$. Take $G_{R \uparrow R \uparrow}^{r}\left(x, x^{\prime}\right)$, for example,

$$
\begin{aligned}
G_{R \uparrow R \uparrow}^{r}\left(x, x^{\prime} ; \omega\right) & =\int d t e^{i \omega t} G_{R \uparrow R \uparrow}^{r}\left(x t, x^{\prime} t^{\prime}\right) \\
& =\frac{1}{2 \pi} \int d k \frac{e^{i k\left(x-x^{\prime}\right)}}{\omega-v_{F} k+i \eta} C_{\Lambda}(|k|),
\end{aligned}
$$

where $\Lambda$ is the cutoff of the momentum and $C_{\Lambda}(|k|)$ is the cutting-off function. Since our model is a low-energy approximation, a cutoff of the momentum is necessary and, according to the discussion in the study of graphene, ${ }^{16}$ a sharp cutoff is not suitable here. Therefore, we use a smooth cutting function

$$
C_{\Lambda}(|k|)=e^{-|k| / \Lambda}
$$

With this cutting-off function, we get

$$
G_{R \uparrow R \uparrow}^{r}\left(x, x^{\prime} ; \omega\right)=-\frac{i}{v_{F}} e^{-|\omega| / v_{F} \Lambda} \cdot e^{i\left(\omega / v_{F}\right)\left(x-x^{\prime}\right)} \theta\left(x-x^{\prime}\right),
$$

where $\theta\left(x-x^{\prime}\right)$ is a step function. The retarded Green's function of the left movers is

$$
G_{L \downarrow L \downarrow}^{r}\left(x, x^{\prime} ; \omega\right)=\frac{i}{v_{F}} e^{-|\omega| / v_{F} \Lambda} e^{i\left(\omega / v_{F}\right)\left(x^{\prime}-x\right)} \theta\left(x^{\prime}-x\right) .
$$

Here, $e^{-|\omega| / v_{F} \Lambda}$ is the decay factor of the retarded Green's function. We have to emphasize that the Green's functions with different smooth cutting functions will have similar form. The only difference is the decay factor.

Then substituting the Green's functions into Eq. (3), we will get the final expression of the exchange interaction between two local spins. In the calculation, we set $R_{12}=x_{1}-x_{2}>0$, and then

$$
\begin{aligned}
E_{R K}= & -\frac{J^{2}}{\pi} \operatorname{Im} \int_{-\infty}^{E_{F}} d \omega \\
& \times\left[\left(S_{1} \cdot \sigma\right)_{\downarrow \uparrow} G_{R \uparrow R \uparrow}^{r}\left(R_{12}\right)\left(S_{2} \cdot \sigma\right)_{\uparrow \downarrow} G_{L \downarrow L \downarrow}^{r}\left(-R_{12}\right)\right] \\
= & -\frac{J^{2}}{\pi} \operatorname{Im} \int_{-\infty}^{E_{F}} d \omega
\end{aligned}
$$




$$
F_{2}\left(R_{12}\right)=-\frac{J^{2}}{\pi} \operatorname{Im}\left[i \int_{-\infty}^{E_{F}} d \omega G_{R \uparrow R \uparrow}^{r}\left(R_{12}\right) G_{L \downarrow L \downarrow}^{r}\left(-R_{12}\right)\right],
$$

and that for term of $S_{1 x} S_{2 y}$ is

$$
F_{3}\left(R_{12}\right)=-F_{2}\left(R_{12}\right) .
$$

Hence, we only need to consider the range functions $F_{1}\left(R_{12}\right)$ and $F_{2}\left(R_{12}\right)$.

We see that all the range functions are related to the kernel function

$$
K(R)=\int_{-\infty}^{E_{F}} d \omega G_{R \uparrow R \uparrow}^{r}(R) G_{L \downarrow L \downarrow}^{r}(-R) .
$$

It depends on the Fermi energy

$K(R)$

$$
= \begin{cases}\frac{\exp \left[\frac{2 E_{F}}{v_{F}}\left(\frac{1}{\Lambda}+i R\right)\right]}{2 v_{F}\left(\frac{1}{\Lambda}+i R\right)}, & E_{F} \leq 0 \\ \frac{1}{2 v_{F}}\left\{\frac{\frac{2}{\Lambda}}{\frac{1}{\Lambda^{2}}+R^{2}}-\frac{\exp \left[-\frac{2 E_{F}}{v_{F}}\left(\frac{1}{\Lambda}-i R\right)\right]}{\frac{1}{\Lambda}-i R}\right\}, & E_{F}>0 .\end{cases}
$$

Finally, for cases $E_{F} \leq 0$, we get the range functions

$$
\begin{gathered}
F_{1}(R)=-\frac{J^{2} e^{2 E_{F} / v_{F} \Lambda}}{2 \pi v_{F}\left(\frac{1}{\Lambda^{2}}+R^{2}\right)}\left[\frac{\sin \left(\frac{2 E_{F} R}{v_{F}}\right)}{\Lambda}-R \cos \left(\frac{2 E_{F} R}{v_{F}}\right)\right], \\
F_{2}(R)=-\frac{J^{2} e^{2 E_{F} / v_{F} \Lambda}}{2 \pi v_{F}\left(\frac{1}{\Lambda^{2}}+R^{2}\right)}\left[\frac{\cos \left(\frac{2 E_{F} R}{v_{F}}\right)}{\Lambda}+R \sin \left(\frac{2 E_{F} R}{v_{F}}\right)\right],
\end{gathered}
$$

and for cases $E_{F}>0$,

$$
\begin{aligned}
F_{1}(R)= & -\frac{J^{2} e^{2\left(-E_{F}\right) / v_{F} \Lambda}}{2 \pi v_{f}\left(\frac{1}{\Lambda^{2}}+R^{2}\right)} \\
& \times\left\{\frac{\sin \left[\frac{2\left(-E_{F}\right) R}{v_{F}}\right]}{\Lambda}-R \cos \left[\frac{2\left(-E_{F}\right) R}{v_{F}}\right]\right\},
\end{aligned}
$$

$$
\begin{aligned}
F_{2}(R)= & -\frac{J^{2}}{2 \pi v_{F}\left(\frac{1}{\Lambda^{2}}+R^{2}\right)} \\
& \times\left\{\frac{2}{\Lambda}-e^{-2 E_{F} / v_{F} \Lambda}\left[\frac{\cos \left(\frac{2 E_{F} R}{v_{F}}\right)}{\Lambda}-R \sin \left(\frac{2 E_{F} R}{v_{F}}\right)\right]\right\} .
\end{aligned}
$$

\section{DISCUSSION}

In the continuum limit $\Lambda\left|x-x^{\prime}\right|=\infty$, we will get a rather simple expression

$$
\begin{aligned}
E_{R K}= & \frac{J^{2}}{2 \pi v_{F}|R|}\left[\cos \alpha\left(S_{1 x} S_{2 x}+S_{1 y} S_{2 y}\right)\right. \\
& \left.-\sin \alpha\left(S_{1 y} S_{2 x}-S_{1 x} S_{2 y}\right)\right],
\end{aligned}
$$

where $R=x-x^{\prime}$ is the distance between the two local spins and $\alpha=\frac{2|R| E_{F}}{v_{F}}$.

If we consider the two local spins $S_{1}$ and $S_{2}$ as classical spins, Eq. (19) can be transformed into

$$
E_{R K}=\frac{J^{2} M^{2}}{2 \pi v_{F}|R|} \sin \theta_{1} \sin \theta_{2} \cos \left[\left(\phi_{1}-\phi_{2}\right)+\alpha\right] .
$$

Here, $\left(M, \theta_{1}, \phi_{1}\right)$ and $\left(M, \theta_{2}, \phi_{2}\right)$ are the spherical coordinates of the spin vectors $S_{1}$ and $S_{2}$. We can see that only if $\theta_{1}=\theta_{2}=\pi / 2$ and $\cos \left[\left(\phi_{1}-\phi_{2}\right)+\alpha\right]=-1$, the system will have its lowest energy. In this case, $\theta_{1}=\theta_{2}=\pi / 2$ means that $S_{1}$ and $S_{2}$ are in plane, and $\cos \left[\left(\phi_{1}-\phi_{2}\right)+\alpha\right]=-1$ shows that the exchange interaction is noncollinear. It means that the effective exchange interaction mediated by the helical edge states is an in-plane and noncollinear coupling. Actually, it is easy to see from the expressions that in plane and noncollinear are the general characteristics of the helical edge states mediated exchange interaction, which does not depend on the continuum limit.

In the continuum limit, the angle between the local spins is determined by $\alpha=\frac{2|R| E_{F}}{v_{F}}$, i.e., the Fermi energy $E_{F}$ and the distance $R$. Especially when the Fermi energy $E_{F}=0$, i.e., the Fermi level is around the Dirac point $\alpha=0$ and $\phi_{1}-\phi_{2}=\pi$. It means that the exchange coupling becomes a constant and is always antiferromagnetic. Here, the Dirac point is the crossing of the bands of right and left movers. However, in general cases, we do not have a simple formula about the angle between the local spins. It should be determined though concrete numerical calculation.

The special characteristics of the helical edge states mediated exchange interaction result from the interplay between the helicity and the linear band dispersion. As shown in former studies, without helicity, if the system is spin independent, the coupling will have similar form $F(R) S_{1} S_{2}$ and the only difference is just the range function $F(R)$. In our case, the helicity makes the system spin dependent: though the matrix of Green's function is still diagonal but $G_{R \uparrow R \uparrow}^{r}\left(x, x^{\prime} ; \omega\right) \neq G_{L \downarrow L \downarrow}^{r}\left(x, x^{\prime} ; \omega\right)$. It is the main reason of the 
noncollinear behavior of the exchange coupling. In addition to the helicity, the linear band dispersion induces opposite step functions into the Green's functions, as shown in Eqs. (7) and (8). Actually, the in-plane characteristic of the exchange interaction mainly results from these step functions.

\section{CONCLUSION}

In summary, based on the simplified model of the helical edge states, we have investigated the helical edge states that mediate RKKY interaction between local spins in the QSH system. Since the conducting electrons in the helical edge states are the only carriers in the QSH insulator, this exchange interaction is probably the only possible mechanism of the exchange coupling between local spins in such a promising spintronic system. Furthermore, due to the helicity, i.e., the correlation between the spin polarization and propagation, it is believed that pure spin current can be realized in this edge state. It means that this exchange interaction is actually an exchange interaction mediated by pure spin current. Hence, this problem is not only of fundamental interest but also useful for the future development of spinbased devices in such systems.

We analyze the simplest theoretical model of the helical edge states and concentrate on the effects of the helicity and its linear band dispersion. We find that the RKKY interaction mediated by this helical edge state in such a system is in plane and noncollinear, which is extremely different from the exchange interaction in other systems. The angle between the local spins depends on the Fermi energy of the system.
Therefore, this effective interaction offers a possible way to control such angle through adjusting the Fermi level by a gate. In the continuum limit, a simple expression of this exchange interaction can be achieved. Especially, when the Fermi level is around the Dirac point, the exchange coupling becomes a constant antiferromagnetic one. We also point out that these peculiar properties result from the interplay between the helicity and the linear band dispersion.

However, our study is based on the noninteracting lowenergy approximation model of the helical edge states. The assumption that the spins of the helical edge modes are pointing along $z$ direction is a good approximation for the Kane-Mele model ${ }^{1}$ (graphene) and the strained semiconductor. 5 The BHZ model ${ }^{2}$ (HgTe/CdTe quantum well) is more complex and more practical factors should be included. As far as we know, the spin orientation of the helical edge modes is still unknown. A more realistic and complicated theoretical model has to be used in order to investigate the RKKY interaction in such system. It is just the project we are working on. Our analysis in this work is actually a good starting point for further investigation.

Finally, we propose that the magnetic impurities deposited on the QSH thin film ${ }^{24}$ is an ideal system to experimentally investigate the helical edge state mediated RKKY interaction with scanning tunneling microscopy.

\section{ACKNOWLEDGMENTS}

We wish to acknowledge the partial support from RGC Grant of HKSAR. X.C.X is supported by DOE and C-Spin Center of Oklahoma.
${ }^{1}$ C. L. Kane and E. J. Mele, Phys. Rev. Lett. 95, 226801 (2005).

${ }^{2}$ B. A. Bernevig, T. L. Hughes, and S. C. Zhang, Science 314, 1757 (2006).

${ }^{3}$ M. Konig, S. Wiedmann, C. Brune, A. Roth, H. Buhmann, and L. W. Molenkamp, X.-L. Qi, and S. C. Zhang, Science 318, 766 (2007).

${ }^{4}$ C. Wu, B. A. Bernevig, and S. C. Zhang, Phys. Rev. Lett. 96, 106401 (2006).

${ }^{5}$ B. A. Bernevig and S. C. Zhang, Phys. Rev. Lett. 96, 106802 (2006).

${ }^{6}$ L. Sheng, D. N. Sheng, C. S. Ting, and F. D. M. Haldane, Phys. Rev. Lett. 95, 136602 (2005).

${ }^{7}$ C. Kittel, in Solid State Physics, edited by F. Seitz, D. Turnbull, and H. Ehrenreich (Academic, New York, 1968), Vol. 22, p. 1.

${ }^{8}$ S. S. P. Parkin, N. More, and K. P. Roche, Phys. Rev. Lett. 64, 2304 (1990).

${ }^{9}$ T. Dietl, H. Ohno, J. Cibert, and D. Ferrand, Science 287, 1019 (2000).

${ }^{10}$ L. I. Glazman and R. C. Ashoori, Science 304, 524 (2004).

${ }^{11}$ N. J. Craig, J. M. Taylor, E. A. Lester, C. M. Marcus, M. P. Hanson, and A. C. Gossard, Science 304, 565 (2004); P. Simon, R. Lopez, and Y. Oreg, Phys. Rev. Lett. 94, 086602 (2005).

${ }^{12}$ H. Imamura, P. Bruno, and Y. Utsumi, Phys. Rev. B 69, 121303(R) (2004).

${ }^{13}$ P. Lyu, N. N. Liu, and C. Zhao, J. Appl. Phys. 102, 103910
(2007).

${ }^{14}$ H.-H. Lai, W.-M. Huang, and H.-H. Lin, Phys. Rev. B 79, 045315 (2009).

${ }^{15}$ L. Brey, H. A. Fertig, and S. Das Sarma, Phys. Rev. Lett. 99, 116802 (2007)

${ }^{16}$ S. Saremi, Phys. Rev. B 76, 184430 (2007).

${ }^{17}$ M. A. H. Vozmediano, M. P. Lopez-Sancho, T. Stauber, and F. Guinea, Phys. Rev. B 72, 155121 (2005).

${ }^{18}$ V. K. Dugaev, V. I. Litvinov, and J. Barnas, Phys. Rev. B 74, 224438 (2006).

${ }^{19}$ C.-Y. Hou, E.-A. Kim, and C. Chamon, Phys. Rev. Lett. 102, 076602 (2009).

${ }^{20}$ A. Ström and H. Johannesson, Phys. Rev. Lett. 102, 096806 (2009).

${ }^{21}$ H. Jiang, S. G. Cheng, Q.-F. Sun, and X. C. Xie, Phys. Rev. Lett. 103, 036803 (2009).

${ }^{22}$ J. Maciejko, C. Liu, Y. Oreg, X.-L. Qi, C. Wu, and S. C. Zhang, Phys. Rev. Lett. 102, 256803 (2009).

${ }^{23}$ N. F. Schwabe, R. J. Elliott, and N. S. Wingreen, Phys. Rev. B 54, 12953 (1996).

${ }^{24}$ C. Liu, B. Yan, X. Qi, T. Frauenheim, X. Dai, Z. Fang, and S. Zhang, arXiv:0908.3654 (unpublished); H. Lu, W. Shan, W. Yao, Q. Niu, and S. Shen, arXiv:0908.3120 (unpublished); J. Linder, T. Yokoyama, and A. Sudbo, arXiv:0908.2992, Phys. Rev. B (to be published). 